\title{
Nanoengineered $\mathrm{Gd}_{3} \mathrm{Al}_{2} \mathrm{Ga}_{3} \mathrm{O}_{12}$ Scintillation Materials with Disordered Garnet Structure for Novel Detectors of lonizing Radiation
}

\author{
Mikhail Korzhik, * Vladimir Alenkov, Oleg Buzanov, Andrei Fedorov, Georgy \\ Dosovitskiy, Larisa Grigorjeva, Vitaliy Mechinsky, Peter Sokolov, Yauhen \\ Tratsiak, Aleksejs Zolotarjovs, Valery Dormenev, Aleksei Dosovitskiy, Devesh \\ Agrawal, Toyli Anniyev, Maxim Vasilyev, and Valery Khabashesku*
}

\section{Introduction}

the last decade, tremendous progress in the improvement of scintil-lation properties was High stopping power, high scintillation yield,dmingmestratgd frosmixed inorganic scintillation crystals. "2] In this case, "mixing" means olution, and minimal levels of afterglow represembainimgicsdverabisovalent cations or anions in the compound without changing its space parameters the scintillation materials must posseysmedteyseflectednsequence of mixing is the disordering of the crystal structure, which for applications in industry, medicine, and science. Inalddwistiba,introduction of several positive features into the crystalline multi-functionality, particularly the ability to detect gammaquanta and neutrons, becomes a matter of elevated interest, system.

Increasing the lasing transitions bandwidth in the mixed crystals of the gar-net structure has been exploited for

generating ultra-short laser pulses. ${ }^{[3]}$ This process is enabled by the inhomogeneous broadening of $4 \mathrm{f}$ spectral lines of the activator ions, which are peculiar to the mixed crystals but absent in the binary garnets. Broadening of the $4 \mathrm{f}-4 \mathrm{f}$ transitions evidently confirms that even a random distribution of cations in the lattice results in considerable distortion of the lattice. The distortion results in several different spatially nonequivalent positions in the garnet-type lattice for the localization of trivalent iron group and rare-earth dopant ions. ${ }^{[4-6]}$ 
Table 1. Properties of some scintillation materials.

\begin{tabular}{|c|c|c|c|c|c|}
\hline Material & $\begin{array}{l}\text { Density } \\
{\left[\mathrm{g} \mathrm{cm}^{-3}\right]}\end{array}$ & $<_{\text {eff }}$ & $\begin{array}{c}\text { Yield } \\
\text { [photons per MeV] }\end{array}$ & $I \mathrm{sc}[\mathrm{ns}]$ & $\lambda_{\mathrm{sc}}[\mathrm{nm}]$ \\
\hline $\mathrm{Nal}(\mathrm{TI})$ & 3.67 & 51 & 43000 & 230 & 415 \\
\hline $\mathrm{Csl}(\mathrm{TI})$ & 4.51 & 54 & 51800 & 1000 & 560 \\
\hline Вi3Ge4O12 (BGO) & 7.13 & 75 & 8200 & 300 & 505 \\
\hline Lu3Al5O12:Pr & 6.7 & 63 & 16000 & 26 & 308 \\
\hline LuAlO3:Ce (LuAP) & 8.34 & 65 & 11400 & $17+$ slow & 365 \\
\hline Lu2SiO5:Ce (LSO) & 7.4 & 66 & 27000 & 40 & 420 \\
\hline $\mathrm{CeBr3}$ & 5.2 & 56 & 58000 & 21 & 360380 \\
\hline LaBr3:Ce (B380) & 5.1 & 47 & 73000 & 30 & 375 \\
\hline $\mathrm{Gd}_{3} \mathrm{Al}_{2} \mathrm{Ga}_{3} \mathrm{O}_{12} \mathrm{Ce}$ & 6.7 & 51 & 46000 & 80200 & 520 \\
\hline
\end{tabular}

Disordering increases the light yield from Ce-doped scintillation material because of the improved conversion efficiency. The modification of the local structure of mixed crystals introduces micro-nonuniformity, reducing the thermalization and diffusion lengths of carriers. Local nonuniformity causes local spatial fluctuations of the band gap in dielectrics, enhancing the ability for recombination of geminate pairs. ${ }^{[/]}$

The mixture of cations changes the band gap energy in solid solutions. ${ }^{[8]}$ Such a change was observed in $\left(\mathrm{Lu}_{1-\mathrm{x}} \mathrm{Y}_{\mathrm{x}}\right) \mathrm{AlO}_{3}: \mathrm{Ce}$, lutetium-gadolinium oxy-orthosilicate, and many other mixed oxides. ${ }^{[9]}$ The shift of the bottom of the conduction band can cover some shallow traps located below the bottom of this band and diminish the thermo-activation energy of deep traps as well. ${ }^{[10]}$ As a consequence, the shift can minimize the loss of scintillation efficiency due to the carriers capture.

Table 1 shows a comparison of properties of some scintillation crystals obtained from single cation, binary, and mixed systems with $\mathrm{NaI}(\mathrm{Tl})$ and $\mathrm{Bi}_{4} \mathrm{Ge}_{3} \mathrm{O}_{12}$ (BGO) crystals. These crystals are widely used and are prospects for application in wireline logging tools.

The mixed crystals of garnet structures demonstrate spectacular progress in improved scintillation properties. ${ }^{[2]}$ Particularly interesting are mixed garnets, which exhibit a high light yield of up to 70000 photons $\mathrm{MeV}^{-1}$, have a luminescence decay time shorter than $100 \mathrm{~ns}$, and emission band peaks at $520 \mathrm{~nm}$, which perfectly match the sensitivity spectrum of the conventional Silicon Photo-Multipliers (SiPMs). ${ }^{[1]}$ Novel gadolinium containing scintillation materials can become the scintillators of choice in high-resolution $\gamma$-radiation spectrometry and compete with halide scintillators recently developed for this purpose. ${ }^{[12]}$

Finally, natural gadolinium is a mixture of six stable isotopes, ${ }^{154} \mathrm{Gd}(2.18 \%),{ }^{155} \mathrm{Gd}(14.8 \%),{ }^{156} \mathrm{Gd}(20.5 \%),{ }^{157} \mathrm{Gd}(15.7 \%),{ }^{158} \mathrm{Gd}$ (24.8\%), and ${ }^{160} \mathrm{Gd}(21.9 \%)$, two of which, ${ }^{155} \mathrm{Gd}$ and ${ }^{157} \mathrm{Gd}$, have the highest cross section of neutron capture of all known stable
However, along with the advantages caused by disordering, a modification of defects occurs in mixed crystals, and new trap centers appear. Although new trap centers can dramatically deteriorate the scintillation properties due to the transformation of scintillation into phosphorescence, they can also introduce new features that become suitable for other applications. For instance, mixed $\mathrm{Al} / \mathrm{Ga}$ garnets present a good opportunity for creation of phosphors with persistent luminescence. ${ }^{[14]}$ In addition, a strong persistent luminescence is obtained in the samples of $\mathrm{Y}_{3} \mathrm{Al}_{2} \mathrm{Ga}_{3} \mathrm{O}_{12}$ :Ce ceramics co-activated with chromium ions. ${ }^{[1 \mathrm{~J}]}$ However, the presence of strong phosphorescence raises serious questions about the mass use of $\mathrm{Al} / \mathrm{Ga}$ mixed garnets for scintillation detectors.

Suppression of the phosphorescence mechanism in $\mathrm{Al} / \mathrm{Ga}$ mixed garnets, particularly in $\mathrm{Gd}_{3} \mathrm{Al}_{2} \mathrm{Ga}_{3} \mathrm{O}_{12}$ : $\mathrm{Ce}$ (GAGG), becomes a crucial task for making this crystal available for wide ap-

plication. The presence of phosphorescence is not sensitive to the kind of the scintillator production technology and purity of raw material. Strong phosphorescence is observed in single crystals and ceramic samples of mixed garnets $\mathrm{Gd}_{3} \mathrm{Al}_{2} \mathrm{Ga}_{3} \mathrm{O}_{12}$ : $\mathrm{Ce}$, made

from raw material with a purity better than $99.995 \%$ and doped solely with Ce ions. ${ }^{[16]}$ It occurs under the excitation in a wide

spectral range including all intra-center excitation bands of $\mathrm{Ce}^{3+}$.

Obviously, phosphorescence in a GAGG crystal is caused by intrinsic defects of the host crystal structure, but the role and contribution of different kinds of defects into this process has not been clarified.

This article presents further improvements of key production technology aspects for $\mathrm{Gd}_{3} \mathrm{Al}_{2} \mathrm{Ga}_{3} \mathrm{O}_{12}$ :Ce single crystals to make them available for a wireline logging tools. At present, no technological problems exist for obtaining large GAGG ingots. ${ }^{[1 /]}$ Con-

sequently, the replacement of BGO by GAGG seems technically possible. However, to scale up the process from a quality sample with the volume of just a few cubic centimeters to a high-quality scintillation element with the dimensions of at least 3 inches in diameter by 4 inches in length, several generic and technological matters must be clarified. These are the origin of the point structure defects in the mixed crystal, and a set of technological improvements to minimize the influence of defects on the scintillation in the material.

\section{Measurements}

A scanning electron microscope (SEM) "JSM-7800F" was used for energy-dispersive spectroscopy (EDS) chemical element mapping of the surface of the samples. The length of each linear scan was $500 \mathrm{~nm}$ with 436 data points collected. The same instrument 
isotopes: 61000 and 254000 barns, respectively. The capture of neutrons is accompanied by the emission of $\gamma$-quanta with the total energy of approximately $8 \mathrm{MeV}$ :

$$
\begin{gathered}
\mathrm{n}+{ }_{\mathrm{n}}+{ }^{157} \mathrm{Gd} \rightarrow{ }^{156} \mathrm{Gd} \rightarrow{ }^{158} \mathrm{Gd}+\gamma(8.5 \mathrm{MeV} \\
\end{gathered}
$$

Individual $\gamma$-quanta can be detected by the same crystal in which the interaction takes place, as shown in ref. [13]. was used for SEM imaging.

In thermally stimulated luminescence (TSL) experiments, performed on a custom-made laboratory bench, the fractional glow method $^{[18]}$ was applied to define the thermal activation energy of the traps ETA. The TSL peaks were measured in the 300 to $800 \mathrm{~nm}$ luminescence spectral range at the heating rate of $6 \mathrm{~K}$ $\mathrm{min}^{-1}$. The samples were activated for $30 \mathrm{~min}$ using an X-ray tube $(30 \mathrm{kV}, 15 \mathrm{~mA})$ at temperature close to that of liquid helium. The spectra of TSL were detected using the "Andor Shamrock B303i" spectrometer with a CCD camera "DU-401A-BV". 
For room-temperature measurements of pulse height spec-tra of laboratory sources of $\gamma$-quanta ${ }^{137} \mathrm{Cs}$ and neutrons $\mathrm{Am}-\mathrm{Be}$, a setup based on a "Philips XP 2262" photomultiplier (PMT) and "ORTEC TRUMP 2k PCI" multi-channel analyzer (MCA) was employed. Spectral data processing was performed with the "ROOT v.5.26" software package. The light yield was measured by an "XP2262 PMT" tube calibrated by using a 1 inch $\mathrm{CsI}(\mathrm{Tl})$ reference crystal produced by ISMA (Kharkov, Ukraine).

The scintillation kinetics were measured at room tempera-ture on the instrumentation bench assembled from the source of annihilation gamma-quanta ${ }^{22} \mathrm{Na}$ and a two-channel measur-ing unit comprised of "start" channel based on a CsF scintillation crystal and a fast time "XP2020" PMT made by Photonis, and a "stop" channel using an identical PMT.

In this bench, timing of the PMT's signals was carried out by two constant fraction discriminators (CFDs), the signals from which arrived at the time-to-amplitude converter (TAC), which converted the difference in arrival times of the "start" and "stop" signals into a pulse of the output voltage with an amplitude proportional to this difference. Pulses from TAC were digitized in an "ORTEC TRUMP 2k PCI" MCA. Acquired kinetics spectra were processed in the "FitKin" software package, where luminescence time constants and their weights (amplitudes) were determined in the three-exponential approximation.

\section{Consideration of Defects in Mixed Crystal Structure}

The difference in the creation of defects in a binary and mixed garnet structure can be recognized from the comparison of the typical binary garnet, yttrium-aluminum garnet $\mathrm{Y}_{3} \mathrm{Al}_{5} \mathrm{O}_{12}$ (YAG), with an appropriate mixed garnet. YAG is a stoichiometric chemical compound, which is a prototype of the garnet family of scintillator materials.

The garnet crystal structure belongs to cubic space group $I A 3 D$ with the cations in spatial positions (on $16 A, 24 C$, and $24 D$ sites) and oxygen anions in the general positions (on $96 \mathrm{H}$ sites). Three cations have tetrahedral coordination (24D sites), and two cations have octahedral ( $16 A$ sites) coordination formed by oxy-gen ions. ${ }^{[19]}$ The eighth coordinate sites (dodecahedral, 24C sites) accommodate yttrium. There are eight formula units in the unit cell of lattice parameter $A 12.01 \mathrm{~A}$ at ambient conditions. The tetrahedra and octahedra are linked to each other by sharing all oxygen corners and to the dodecahedra by shared edges.

Structures of more complex formulations could be obtained from YAG by partially or completely substituting atoms in cationic positions. $^{[20]}$

$\mathrm{Ga}^{3+}$ preferentially occupies a tetrahedral site in YAG and its solid solutions over the octahedral site. For instance, according to Kanai et al., ${ }^{[19]}$ the composition occupancy of $\mathrm{Ga}^{3+}$ ions in $\mathrm{Y}_{3} \mathrm{Al}_{2} \mathrm{Ga}_{3} \mathrm{O}_{12}$ (YAGG) is 0.727 for the tetrahedral sites and 0.409 for the octahedral sites, respectively. In other words, $72.7 \%$ of $\mathrm{Ga}^{3+}$ ions occupy the tetrahedral sites and $27.3 \%$ octahedral sites. Note that in case of random distribution, these values should be $60 \%$ and $40 \%$.

In the gadolinium-gallium-aluminum garnet GGAG, the $\mathrm{Ga}^{3+}$ ion has a substantially greater preference for the tetrahedral site than the $\mathrm{Al}^{3+}$ ion, and this is the same for the YAGG garnet.
The ionic radius of $\mathrm{Gd} \quad$ (1.05 A for VIII-fold coordination) is close to that of $\mathrm{Y}(1.02 \mathrm{~A})$. The ionic radius of $\mathrm{Ce}$ is $1.14 \mathrm{~A}$ for VIII-fold coordination. Doping with the Ce ions usually re-places $\mathrm{Gd}$ in the $24 C$ site. The ionic radius of $\mathrm{Ga}^{3+}(0.62$ and 0.47 A for VI-fold and IV-fold coordination, respectively) is bigger than that of $\mathrm{Al}(0.53$ and $0.39 \mathrm{~A}$ for VI-fold and IV-fold coordination, respectively). The introduction of larger $\mathrm{Ga}^{3+}$ cations into the YAG crystalline matrix distorts initial crystal structure and modifies bond lengths and angles. For example, the cation-oxygen interatomic distance in YAG, 1.754 A and 1.938 A for tetrahedral and octahedral sites, respectively, changes to $1.814 \mathrm{~A}$ and 1.955

$\circ \mathrm{A}$ in YAGG, while the Ga-O-Al bond angle changes from 130.65 to $128.65^{\circ}$.

Both effects, such as the changes in the cation-oxygen interatomic distance and in the bond angle at the conditions of random distribution of the cation, result in considerable lattice strain and distort the polyhedral, forming shallow trapping centers. Large numbers of lattice distortions, which are related to the number of cations in the lattice and moderate the bottom of the conduction zone, become a reason for an increase in the yield of scintillations for mixed crystals according to Belski et al. ${ }^{[1]}$

The alternative model of mixed crystals is represented in ref. [22]by domain structure. These domains, consisting mainly from aluminum and gallium garnets, can be detected by using a linear EDS scan of the surface of the crystal by SEM at high magnification to determine the deviations of $\mathrm{Al}$ and $\mathrm{Ga}$ atomic concentrations from mean values by measurement of intensities of $\mathrm{Ga} \mathrm{L}$ and $\mathrm{Al} \mathrm{K}$ bands.

Figure 1a shows a codependence of deviations / of the intensities of $\mathrm{Ga} \mathrm{L}$ and $\mathrm{Al} \mathrm{K}$ bands on their mean values . Figure $1 \mathrm{~b}$ presents a codependence of the dispersions of intensities of $\mathrm{Ga} \mathrm{L}$ and $\mathrm{Al} \mathrm{K}$ bands on their mean values. The observed codependences of deviations and their dispersions point at homogeneous distribution of gallium and aluminum in the sample as well as lack of segregation of these elements.

The similar observation was made for both as-grown single crystals samples and annealed in the 300-1200 ${ }^{\circ} \mathrm{C}$ temperature range. This can be explained by the development of a defect structure inside the sample that can act as a storage of defects during the process of thermal treatment and preclude a large-distance transfer of mass inside the grains. It can be concluded therefore, that the domain structure is very unlikely to happen in the crys-tals of mixed garnets.

Consequently, we can state that the distortion of the initial crystal structure and modification of bond lengths and angles due to the difference of ionic radii of $\mathrm{Ga}$ and $\mathrm{Al}$ result in formation of intrinsic defects in mixed crystals.

In addition to the defects in $\mathrm{Gd}_{3} \mathrm{Al}_{2} \mathrm{Ga}_{3} \mathrm{O}_{12}$ : Ce caused by random $\mathrm{Ga}$ and $\mathrm{Al}$ distribution, more defects, caused by preferential evaporation of the most volatile component from the melt during crystal growth, are also expected. Slightly oxidizing atmosphere in the crystal growth chamber partly solves this problem, but not completely.

Single crystal growth of multi-element Ga-containing compounds is complicated by the high evaporation rate of $\mathrm{Ga}$ oxide from the melt $\left(\mathrm{Ga}_{2} \mathrm{O}_{3}\right.$ has a maximum vaporization rate that is 4 to 5 orders of magnitude higher than $\mathrm{Al}_{2} \mathrm{O}_{3}$ in the 1600 to $2000{ }^{\circ} \mathrm{C}$ temperature range, see Table 2). Predominant $\mathrm{Ga}$ evaporation makes it difficult to control the crystal composition precisely. 

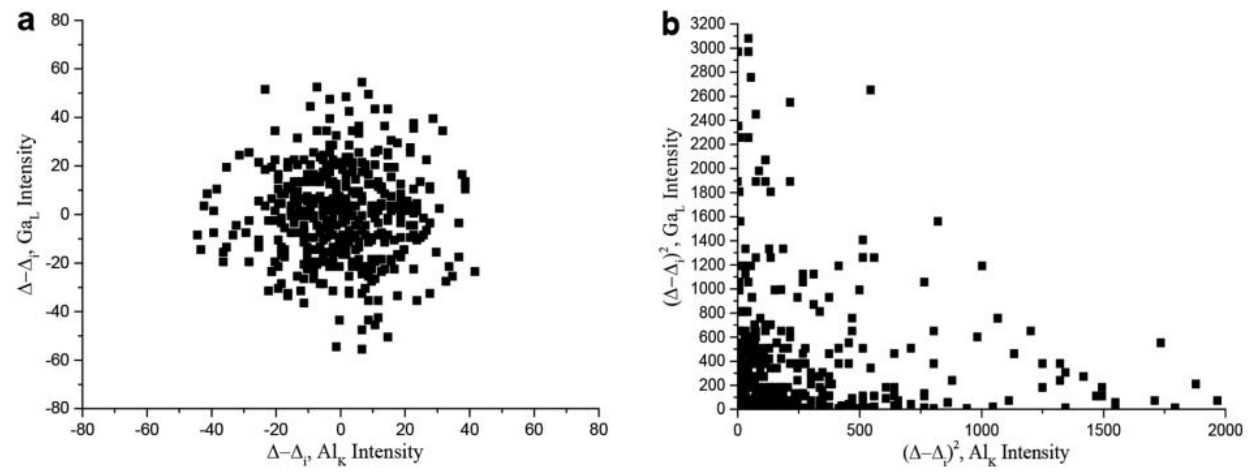

Figure 1. a) Codependence of deviations I of the intensities of $G a L$ and Al K bands on their mean values ; b) codependence of the dispersions of intensities of $\mathrm{Ga} L$ and $\mathrm{Al} K$ bands on their mean values.

Table 2. Maximum vaporization rates for $\mathrm{Ga}$ and $\mathrm{Al}$ oxides at 0.2 bar oxygen partial pressure.

\begin{tabular}{lrcr}
\hline \multirow{2}{*}{ Oxide } & \multicolumn{3}{c}{ Evaporation rate $\left[\mathrm{mol} \mathrm{cm}^{-2} \mathrm{~s}^{-1}\right],[23]$} \\
\cline { 2 - 4 } & $1600{ }^{\circ} \mathrm{C}$ & $1800{ }^{\circ} \mathrm{C}$ & $2000{ }^{\circ} \mathrm{C}$ \\
\hline $\mathrm{Ga}_{2} \mathrm{O}_{3}$ & $8 \times 10^{-8}$ & $4 \times 10^{-5}$ & $5 \times 10^{-3}$ \\
$\mathrm{Al}_{2} \mathrm{O}_{3}$ & $5 \times 10^{-12}$ & $6 \times 10^{-10}$ & $3 \times 10^{-8}$ \\
\hline
\end{tabular}

The formation of cation vacancies due to gallium evapora-tion inevitably leads to the creation of anionic vacancies and additional randomly distributed trapping centers based on such vacancies. These defects are not intrinsic; their presence and concentration are caused by the peculiarities of the technology.

Obviously, different approaches affect the influence of intrin-sic and casually distributed defects.

No technological approach to remove intrinsic defects from the crystal is currently available, but the influence of these defects on the scintillation can be minimized by introducing the mecha-nism to control population of the crystals by nonequilibrium car-riers. A positive role in the GGAG:Ce co-doping with $\mathrm{Mg}^{2+}$ to diminish the level of phosphorescence was demonstrated in. ${ }^{[24}$ Such doping, in which a trivalent ion was substituted by a divalent alkali-earth cation in the host matrix, activated a mechanism of fast recombination of carriers captured by intrinsic defects, and accelerated the luminescence build up in the crystal. ${ }^{[25]}$ However, the co-doping of Ce-activated crystals by divalent ions (even at the level of less than 0.1 atomic \%) oxidized the part of $\mathrm{Ce}^{3+}$ ions into $\mathrm{Ce}^{4+}$. This resulted in a lower scintillation light yield that deteriorated the energy resolution, especially for low-energy gammaquanta. Nevertheless, co-doped samples demonstrated recuperation of the light yield to the level of Ce crystals under the cooling of crystals down to $-45^{\circ} \mathrm{C}$, without any increase of slow component fractions in the scintillation kinetics and phosphorescence appearance. [26] $^{\text {[2] }}$

As for casually distributed defects, their concentration can be minimized at the stage of preparation of raw material.

\section{Preparation of Nanostructured Raw Material}

One possible approach to suppress uneven evaporation of different components is a pre-synthesis of raw material. For this

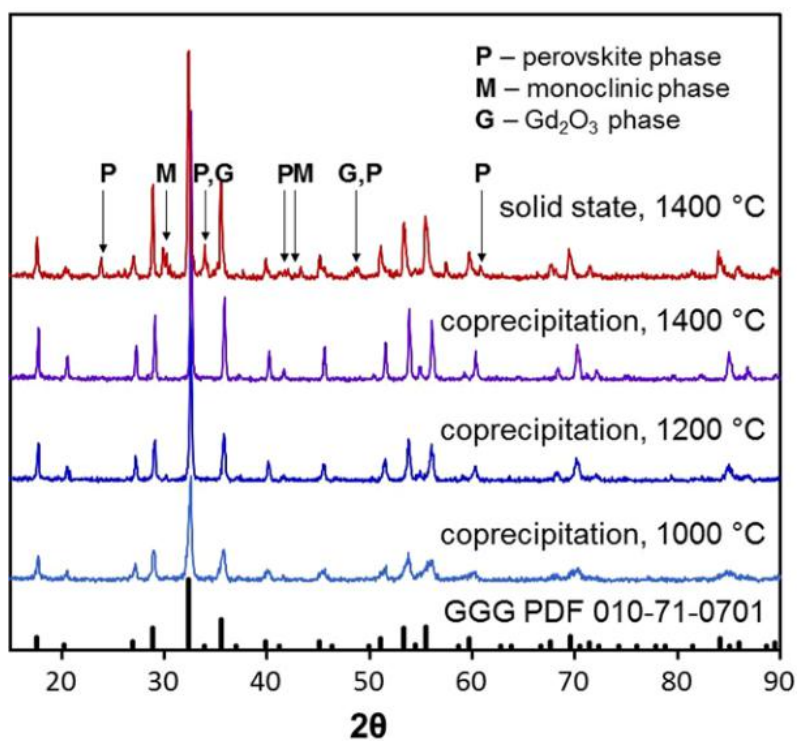

Figure 2. XRD of Gd3Ga3Al2O12:Ce powder obtained by different routes.

purpose, a method of co-precipitation has been considered. This method is used to obtain phosphor powders and nano-powders for making the ceramics, and it could be scaled up to high production volumes. Co-precipitation enables high homogene-ity of components distribution in the product, which facilitates the phase formation at much lower temperatures than for the solid-state reaction. This is demonstrated by the XRD patterns of $\mathrm{Gd}_{3} \mathrm{Ga}_{3} \mathrm{Al}_{2} \mathrm{O}_{12}$ :Ce powders (Figure 2), obtained by different routes. The XRD shows that co-precipitated powder forms garnet phase already after heat treatment at $1000{ }^{\circ} \mathrm{C}$, while the powder, prepared from stoichiometric mixture of oxides by solid-state reaction at $1400{ }^{\circ} \mathrm{C}$, still contains secondary phases of perovskite and monoclinic phases as well as $\mathrm{Gd}_{2} \mathrm{O}_{3}$ phase.

Co-precipitation of GGAG powders involves addition of a water solution, containing all cations mixed in a desired ratio, to a precipitant solution (ammonia was used to obtain powders with dense particles). Next, the precipitate is separated from a mother solution and heat treated to dry it and decompose the precipitated salts to oxides and form the desired phase.

GAGG:Ce powders were obtained by annealing the precursors for $2 \mathrm{~h}$ at $600{ }^{\circ} \mathrm{C}$ and then for $2 \mathrm{~h}$ at $1000{ }^{\circ} \mathrm{C}$ in air. Precursors were 


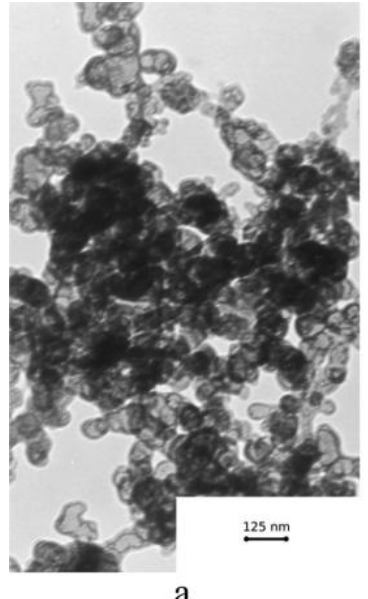

a

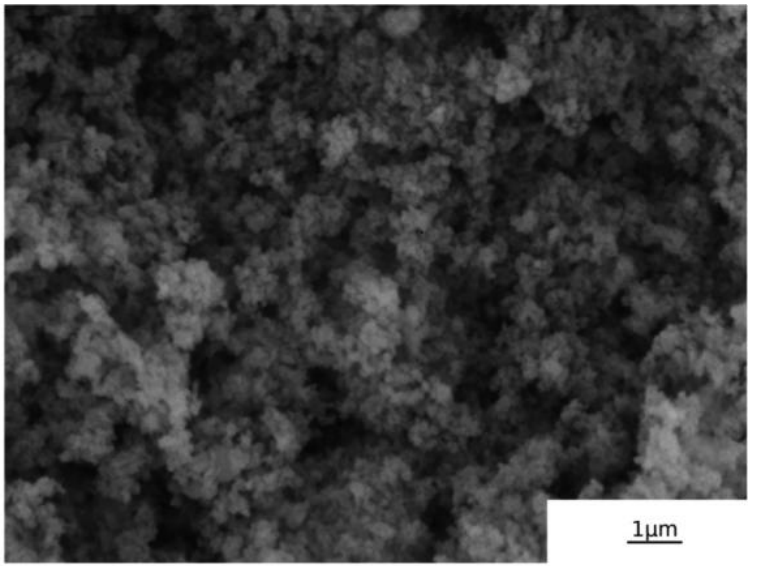

$b$

Figure 3. TEM image of the GAGG: a) Ce precursor specimen and b) SEM image of the powder.

obtained by using a co-precipitation method. $\mathrm{Al}\left(\mathrm{NO}_{3}\right)_{3} \cdot 9 \mathrm{H}_{2} \mathrm{O}$, $\mathrm{Ga}_{2} \mathrm{O}_{3}, \mathrm{Gd}_{2} \mathrm{O}_{3}, \mathrm{Ce}\left(\mathrm{NO}_{3}\right)_{3} \cdot 6 \mathrm{H}_{2} \mathrm{O}, \mathrm{HNO}_{3}, \mathrm{NH}_{4} \mathrm{HCO}_{3}$ reagents were used as raw materials.

As the first step, a $1 \mathrm{~m}$ solution of $\mathrm{Al}\left(\mathrm{NO}_{3}\right)_{3}(\mathrm{pH} 2)$ was prepared by dissolving $\mathrm{Al}\left(\mathrm{NO}_{3}\right)_{3} \cdot 9 \mathrm{H}_{2} \mathrm{O}$ in water acidified by $\mathrm{HNO}_{3}$, while $1 \mathrm{~m}$ water solutions of $\mathrm{Ga}\left(\mathrm{NO}_{3}\right)_{3}$ and $\mathrm{Gd}\left(\mathrm{NO}_{3}\right)_{3}$ were obtained by dissolving a $\mathrm{Ga}_{2} \mathrm{O}_{3}$ and $\mathrm{Gd}_{2} \mathrm{O}_{3}$ in $\mathrm{HNO}_{3}$ solution at boiling. As the next step, the required quantities of $\mathrm{Gd}\left(\mathrm{NO}_{3}\right)_{3}, \mathrm{Ga}\left(\mathrm{NO}_{3}\right)_{3}, \mathrm{Al}\left(\mathrm{NO}_{3}\right)_{3}$, and $\mathrm{Ce}\left(\mathrm{NO}_{3}\right)_{3} \cdot 6 \mathrm{H}_{2} \mathrm{O}$ were mixed by stirring for $30 \mathrm{~min}$ to completely dissolve all components. The resulting transparent solution was dropwise added at intensive stirring to the solution containing a $30 \%$ excess of precipitator $\left(20 \mathrm{~g} \mathrm{NH}_{4} \mathrm{HCO}_{3}\right.$ in $\left.100 \mathrm{~mL} \mathrm{H}_{2} \mathrm{O}\right)$. Stirring was continued for $30 \mathrm{~min}$, and then the obtained white precipitate was isolated and dried at $80{ }^{\circ} \mathrm{C}$ in air.

Electron microscopy images of GAGG:Ce precursor and powder are presented in Figure 3.

TEM was used to determine the particle's size in the GAGG:Ce precursors. The average particle size of the precursor was $60 \mathrm{~nm}$. The concentration of the initial reactants influenced the particles aggregation (which increased with the concentration of the reactants), while the primary particle size did not changed.

After annealing, the average particle size in GAGG:Ce powders became $94 \pm 5 \mathrm{~nm}$. The observed effect of increasing of the particle size may be explained by the following reason: under annealing of the precursor the mass transfer between particles increases, enlarging the particles on one side, and causing parti-cle adhesions from the other side. Increasing the heat treatment temperature amplified these effects.

In addition, solid-state reaction process was used to produce a GAGG raw material from the stoichiometric mixture of oxides. Garnet formation through this process can be described by the following equation:

$3 \mathrm{Gd}_{2} \mathrm{O}_{3}+3 \mathrm{Ga}_{2} \mathrm{O}_{3}+\mathrm{Al}_{2} \mathrm{O}_{3}=2 \mathrm{Gd}_{3} \mathrm{Al}_{2} \mathrm{Ga}_{3} \mathrm{O}_{12}$

In a solid-state method, the process is limited by the diffusion of elements. Consequently, the process requires a high annealing temperature for a longer time to obtain a final compound. The obtained raw material consists of the agglomerated particles with dimensions in the micron range and showing no garnet habitus.
Table 3. Scintillation parameters of studied GAGG samples.

\begin{tabular}{llcc}
\hline Sample & Composition & Raw material & Co-doping \\
\hline SSR1 & Gd3Ga3Al2O12:Ce & Solid state reacted & - \\
SSR2 & Gd3Ga3Al2O12:Ce & Solid state reacted & $\mathrm{Mg}, 10 \mathrm{ppm}$ \\
SSR3 & Gd3Ga3Al2O12:Ce & Solid state reacted & $\mathrm{Mg}, 50 \mathrm{ppm}$ \\
CP1 & Gd3Ga3Al2O12:Ce & Co-precipitated & - \\
CP2 & Gd3Ga3Al2O12:Ce & Co-precipitated & $\mathrm{Mg}, 50 \mathrm{ppm}$ \\
\hline
\end{tabular}

\section{Samples}

The GAGG:Ce samples used in this study were grown by the Czochralski technique in iridium crucibles from two types of the raw material described previously. The samples, in the shape of a $5 \times 5 \times 5 \mathrm{~mm}^{3}$ cubes and $10 \times 10 \times 2 \mathrm{~mm}^{3}$ plates, were cut from single crystal boules and then polished. The compositions of the samples are presented in Table 3. Some of the crystals were codoped by $\mathrm{Mg}$ for the reason described above. All samples were annealed at temperature of $850{ }^{\circ} \mathrm{C}$ for $50 \mathrm{~h}$.

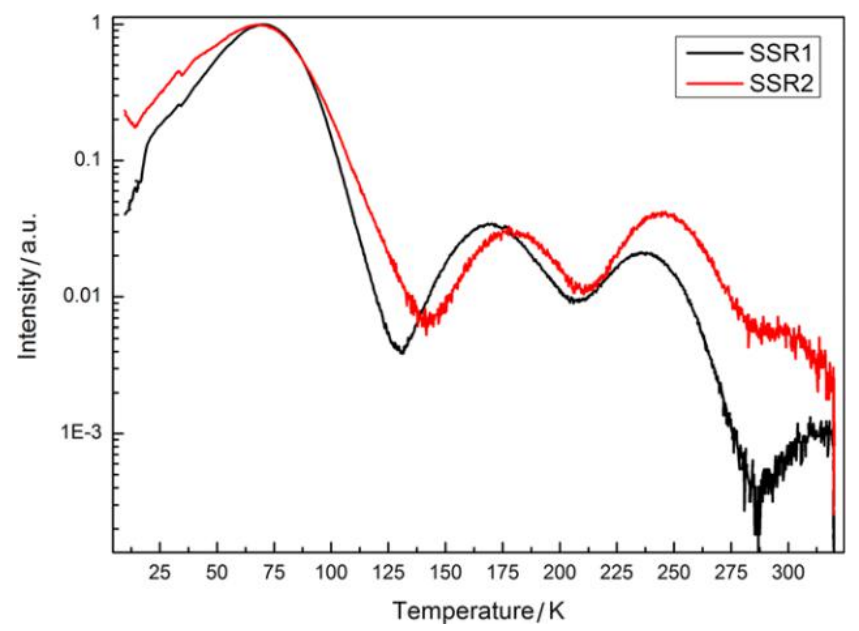

Figure 4. TSL curves of the SSR1 (black) and SSR2 (red) samples. 


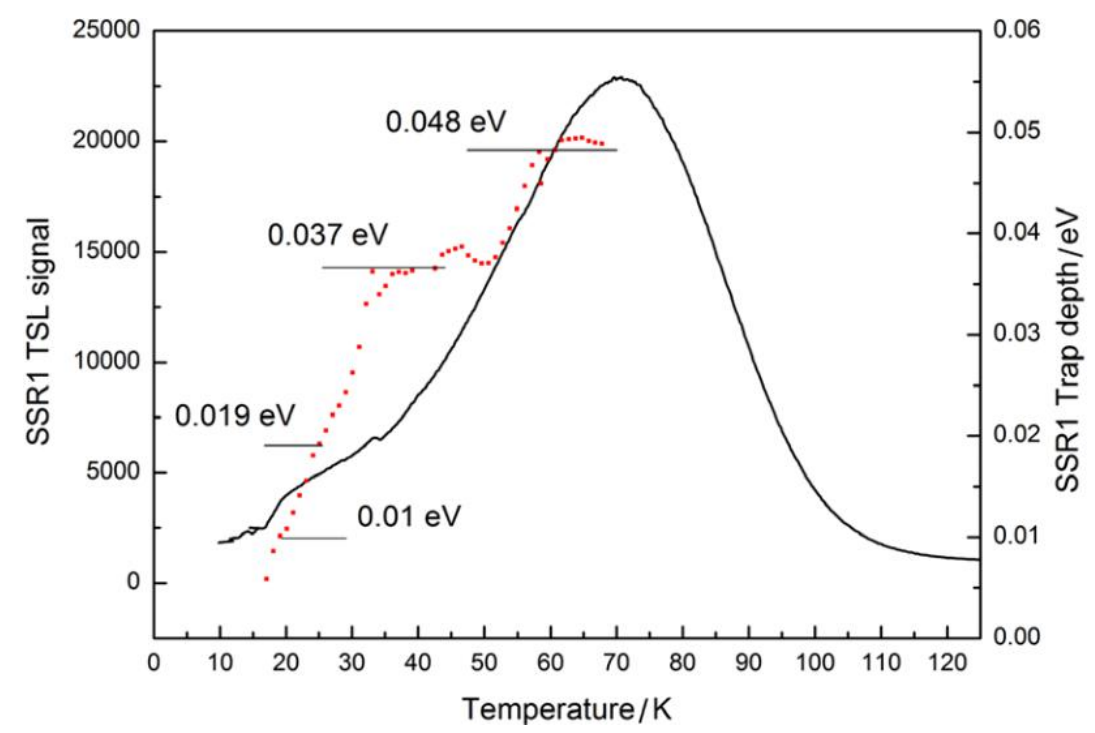

Figure 5. TSL curves (black) and ETA points (red) of SSR1 sample.

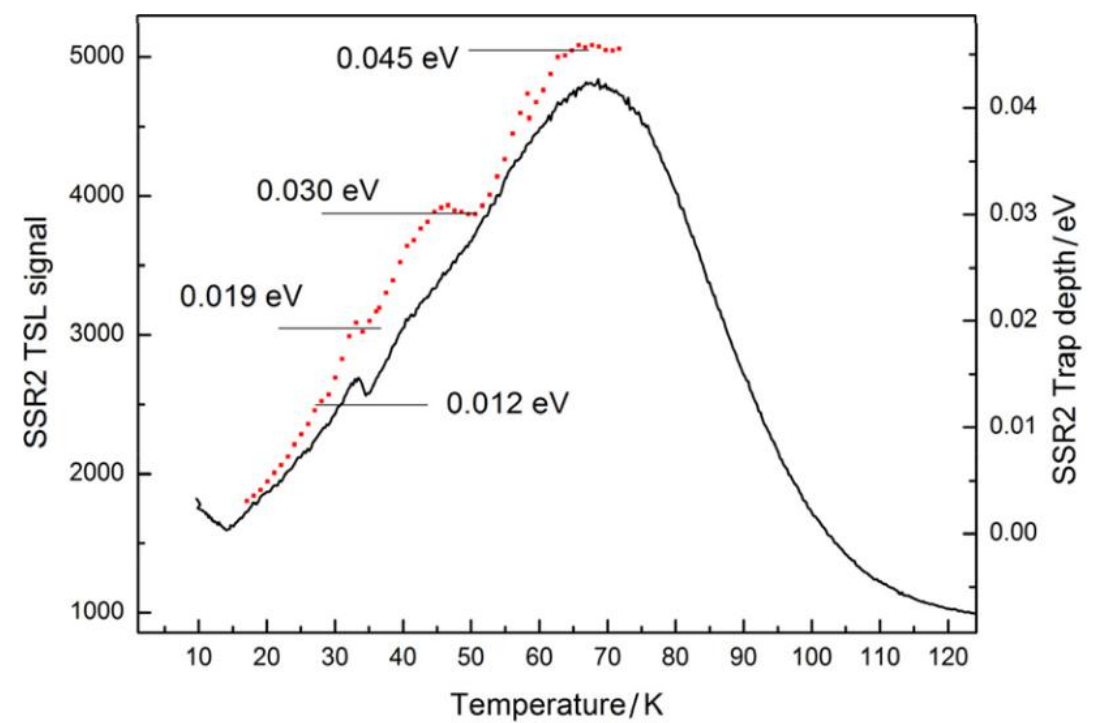

Figure 6. TSL curves (black) and ETA points (red) of SSR2 sample.

\section{Results and Discussion}

TSL is a suitable tool to observe and characterize the trapping centers in the grown crystal. It enables selection of characteristic defects in the crystal that originate from the intrinsic properties of the material and are not influenced by technological condi-tions. Figure 4 shows a TSL spectra of the samples SSR1,2. The spectra of the initiation of TSL glow, absorption spectra of $\mathrm{Ce}^{3+}$ ions and spectra of phosphorescence all coincide, as shown in ref. [27].

The sample, obtained without a co-doping, exhibits strong visually observed room temperature phosphorescence after a stop of the photo-excitation of the absorption bands of $\mathrm{Ce}^{3+}$ ions. Phosphorescence is already strongly suppressed in SSR2 sample, while the TSL intensity decreases its intensity by a factor of about
5. The intensity of a broad peak with the complex structure and a maximum of approximately $75 \mathrm{~K}$ dominates the other bands by a factor of about 50. However, as seen from Figures 5 and 6, its shape is practically not affected by the co-doping with $\mathrm{Mg}$, and its set (different Thermo-Activation energy ETA) of traps remains the same. The same dominating peak is observed for the sample CP1. Weak TSL peaks with $E T A=0.13 \mathrm{eV}(175 \mathrm{~K})$ and $0.18 \mathrm{eV}(250$ $\mathrm{K})$ are also observed in SSR and CP samples. Thus, we concluded that the appearance of the group of low-temperature glow peaks with the maxima near 75,175 , and $250 \mathrm{~K}$ is due to intrin-sic defects caused by shallow traps based on distortions of the crystal structure in the mixed crystal. Figure 7 shows TSL carpet images of CP type samples, where the temperature is given along $\mathrm{Y}$ scale, whereas the wavelength spectrum of TSL is imaged along X scale. 


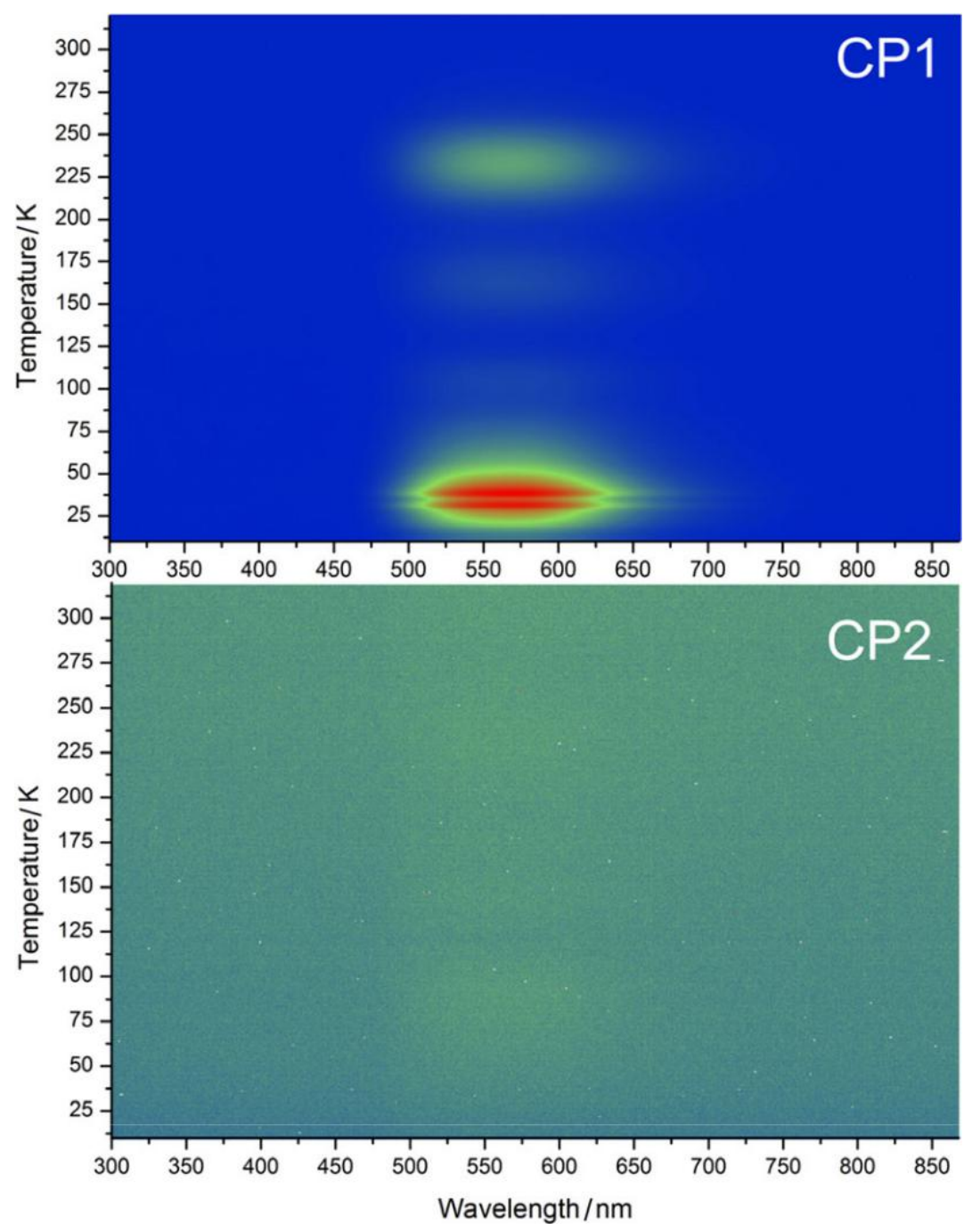

Figure 7. TSL carpet images for the samples CP1 (upper panel) and CP2 (bottom panel).

As seen, only a $\mathrm{Ce}^{3+}$ luminescence is emitted during the thermo-activation of traps. However, in the studied temperature range, TSL is completely suppressed at 50-ppm concentration of $\mathrm{Mg}$ co-doping. The obtained data show that a small concentra-tion of $\mathrm{Mg}$ in GAGG single crystal, at the level of $50 \mathrm{ppm}$, is re-quired for effective prevention of the capture of free carriers by shallow intrinsic traps. In our previous work, ${ }^{[27]}$ we observed that TSL glow peaks located above $300 \mathrm{~K}$ are affected by peculiarities of technology. The spectra show that the TSL of SSR1 also con-tains glow peak above $300 \mathrm{~K}$, whereas a carpet of CP1 does not show any glow in this temperature range. This indicates that coprecipitation of the raw material decreases the concentration of the deep traps in the crystal.

The pulse height spectra of SSR1,2 samples, measured at RT are shown in Figures 8 and 9.

In spite of keeping the sample SSR1 in the dark for one hour before measurements, the spectrum (Figure 8) shows a wide doubled photo-peak. In our opinion, the appearance of such peak is caused by phosphorescence still present in the crystal. Note that even a low concentration of $\mathrm{Mg}$ in the crystal (SSR2) decreases the LY of scintillation by $10 \%$ at room temperature. Moreover, sample SSR3 shows a drop of LY by $40 \%$ as compared to SSR 1 sample.

Unlike SSR series, the CP samples show different behavior. Table 4 shows comparison of the light yield and level of phosphorescence of SSR and CP type samples, measured at room temperature.

Primarily, CP1,2 samples demonstrate better light yield than SSR type samples by $10-20 \%$, which is most likely due to smaller concentration of the vacancy-based defects. Figure 10 shows the spectra of Am-Be source measured with the $18 \times 18 \times 7 \mathrm{~mm}^{3}$ size $\mathrm{Gd}_{3} \mathrm{Al}_{2} \mathrm{Ga}_{3} \mathrm{O}_{12}$ : Ce detector (CP2) at two different thicknesses of boron absorber. Use of the scintillation crystal of smaller size allowed the separation of only main soft bands with the energies near $90 \mathrm{keV}$ (with area S90), $190 \mathrm{keV}$ (S190), and $511 \mathrm{keV}$ (S511) from the total spectra of gamma quanta produced in the reac-tion. It was determined that increase of the thickness of boron absorber from 4.5 to $10.5 \mathrm{~cm}$ led to more than four times de-crease of the S90 area, while the S511 area decreased by only two times. 


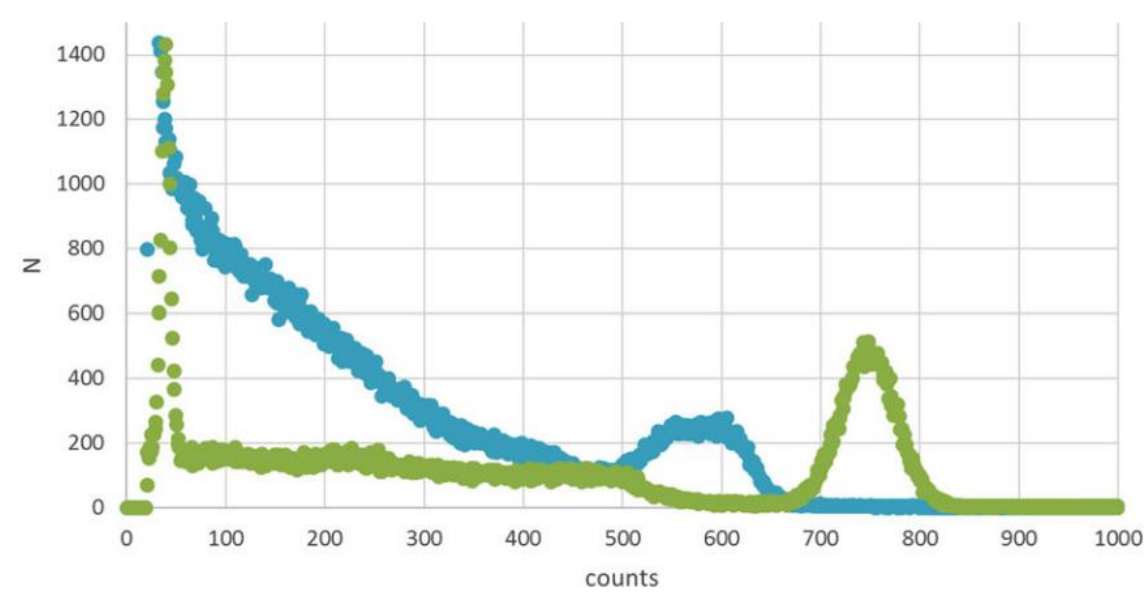

Figure $8 .{ }^{137} \mathrm{Cs}$ pulse height spectra measured at RT with SSR1 sample (blue) and CsI(TI) (green).

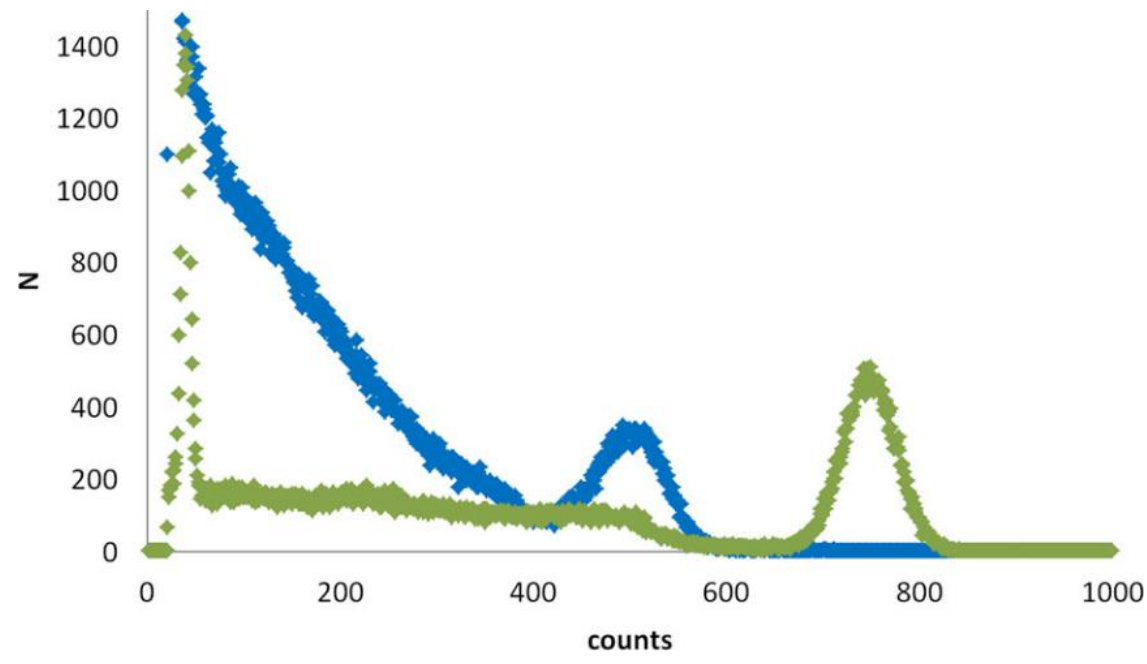

Figure $9 .{ }^{137} \mathrm{Cs}$ pulse height spectra measured at RT with SSR2 sample (blue) and CsI(TI) (green).

Table 4. Comparison of the light yield and phosphorescence level of the SSR and CP type GAGG samples.

\begin{tabular}{lccccc} 
Sample & SSR1 & SSR2 & SSR3 & CP1 & CP2 \\
\hline $\begin{array}{l}\text { Light yield relatively to reference } \\
\text { CsI(TI) [\%] }\end{array}$ & 0.78 & 0.67 & 0.47 & 0.88 & 0.76 \\
$\begin{array}{l}\text { Level of phosphorescence measured } \\
\text { according the method, described } \\
\text { elsewhere }\end{array}$ & 1 & 0.7 & 0.1 & 1 & 0.07 \\
\hline
\end{tabular}

Thus, comparison of the peak parameters of the gamma quanta produced during the process of interaction with the neu-trons in GAGG can provide insights on the parameters of neu-tron source and energy distribution of neutrons in the spectrum.

It is worth noting that the kinetics of scintillation weakly de-pend on the concentration of co-doping in the samples stud-ied. GAGG samples solely doped with $\mathrm{Ce}$ at their percent frac-tions have decay kinetics components close to $30 \mathrm{~ns}(36 \%), 85 \mathrm{~ns}$
(57\%), and $350 \mathrm{~ns}(7 \%)$, whereas Mg-doped samples have slightly shorter decay constants: $27 \mathrm{~ns}(34 \%), 75 \mathrm{~ns}(55 \%), 200 \mathrm{~ns}(11 \%)$. However, a co-doping, as recently shown in, ${ }^{[26]}$ shortens a risetime of $\mathrm{Ce}^{3+}$ luminescence to the ps level, which, in our opinion, will have a positive impact on the coincidence time resolution measured with the co-doped samples.

\section{Conclusions}

The performed study showed that the influence of different types of structure defects in GAGG scintillation crystals doped with Ce ions can be controlled by different technological approaches. The influence of the defects appearing due to disordering of the multiionic structure of the garnet crystal can be controlled by additional aliovalent crystal co-doping by $\mathrm{Mg}$, whereas predomi-nant evaporation of $\mathrm{Ga}$ from the melt during the crystal growth can be $y_{\text {minimized }}$ by utilizing the co-precipitated nanostructured raw material. Use of the co-precipitated raw material promotes 


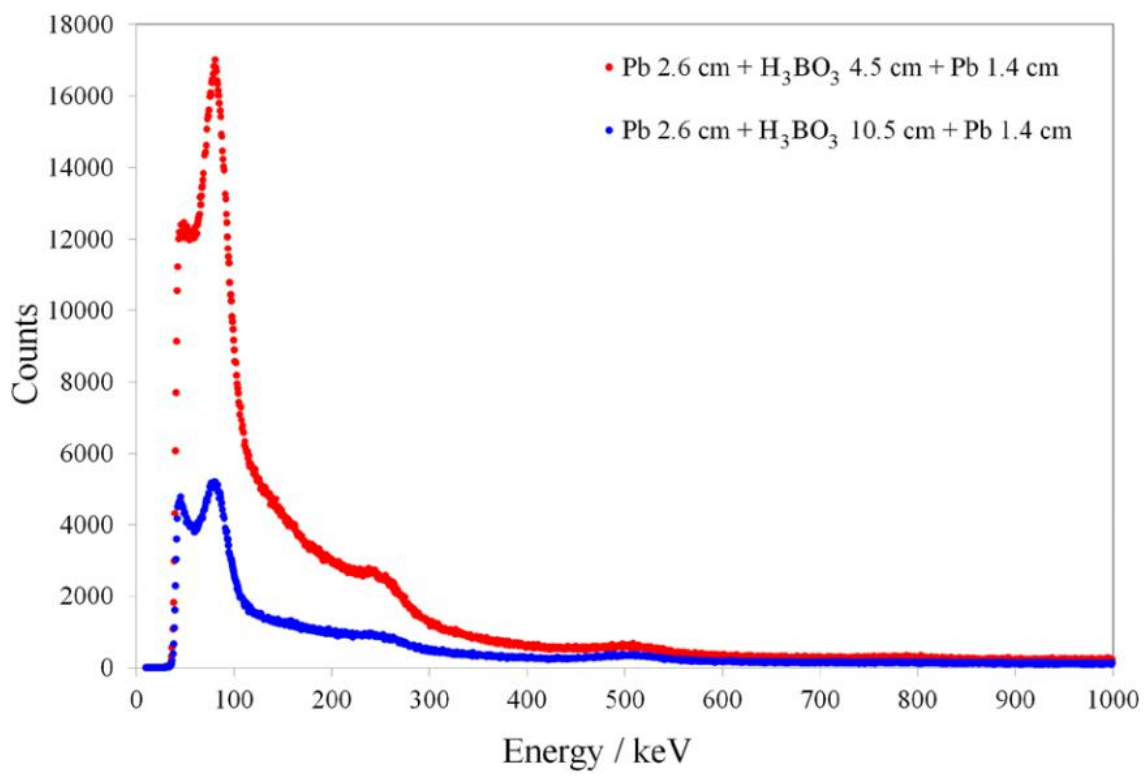

Figure 10. Spectra of Am-Be source measured with the $18 \times 18 \times 7 \mathrm{~mm}^{3}$ size Gd3Al2Ga3O12:Ce detector at two different thicknesses of boron absorber.

an increase of the light yield from such nano-engineered crys-tal by up to $20 \%$. An increase of the light yield of the material makes it useful for neutron detection applications via a relatively soft gamma-quanta created on interaction of neutrons with the nuclei of Gd in the crystal.

\section{Acknowledgements}

The authors are grateful to Baker Hughes a GE Company for support of this activity. This work has also been supported by grant N14.W03.31.0004 from the Government of the Russian Federation.

\section{Conflict of Interest}

The authors declare no conflict of interest.

\section{Keywords}

co-precipitation, disordered crystal, luminescence, multicomponent gar-net, nanoengineering, scintillators

[1] M. Korzhik, A. Gektin, Engineering of Scintillation Materials and Radi-ation Technologies, Springer, Cham, Switzerland 2017.

[2] P. Lecoq, A. Gektin, M. Korzhik, Inorganic Scintillators for Detecting Systems, Springer, Cham, Switzerland 2017.

[3] E. Sorokin, M. H. Ober, I. Sorokina, E. Wintner, A. J. Schmidt, A. I. Zagumennyi, G. B. Loutts, E. W. Zharikov, I. A. Shcherbakov, J. Opt. Soc. Am. B 1993, 10, 1436.
[4] P. Solarz, M. Głowacki, M. Berkowski, W. Ryba-Romanowski, J. Alloys Compd. 2016, 689, 359.

[5] S. Feofilov, A. Kulinkin, K. Ovanesyan, A. Petrosyan, C. Dujardin,Phys. Chem. Chem. Phys. 2014, 16, 22583.

[6] N. M. Khaidukov, V. N. Makhov, Q. Zhang, R. Shi, H. Liang, Dyes Pigm. 2017, 142, 524.

[7] A. V. Gektin, A. N. Belsky, A. N. Vasil'ev, IEEE Trans. Nucl. Sci. 2014, 61, 262.

[8] J. M. Odieglo, Ph.D. Thesis, University of Utrecht, The Netherlands 2012.

[9] J. Mares, M. Nikl, E. Mihokova, A. Beitlerova, A. Vedda, C. D'Ambrosio, IEEE Trans. Nucl. Sci. 2008, 55, 1142.

[10] V. Laguta, Y. Zorenko, V. Gorbenko, A. Iskaliyeva, Y. Zagorodniy, O. Sidletskiy, P. Bilski, A. Twardak, M. Nikl, J. Phys. Chem. C 2016, 120, 24400.

[11] H. L. Kim, H. J. Kim, E. J. Jang, W. G. Lee, M. K. Ki, H. D. Kim, G. S. Jun, V. Kochurikhin, J. Ceram. Process. Res. 2015, 16, 124.

[12] a) $<$ /number $>$ N. J. Cherepy, S. A. Payne, S. J. Asztalos, G. Hull, J. D. Kuntz, T. Niedermayer, S. Pimputkar, J. J. Roberts, R. D. Sanner, T. M. Tillotson, E. Van Loef, C. M. Wilson, K. S. Shah, U. N. Roy, R. Hawrami, A. Burger, L. A. Boatner, W. S. Choong, W. W. Moses, IEEE Trans. Nucl. Sci. 2009, 56, 873; b) Z. Yan, T. Shalapska, E. D. Bourret, J. Cryst. Growth 2016, 435, 42.

[13] V. Dormenev, presented at IEEE 2017 Nuclear Science Symp. and Medical Imaging Conf., Atlanta, GA, October 2017.

[14] J. Ueda, K. Kuroishi, S. Tanabe, Appl. Phys. Express 2014, 7, 062201.

[15] X. Jian, S. Tanabe, A. D. Sontake, J. Ueda, Appl. Phys. Lett. 2015, 107, 081903

[16] G. Dosovitskiy, A. Fedorov, V. Mechinsky, A. Borisevich, A. Dosovit-skiy, E. Tret'jak, M. Korjik, IOP Conf. Ser.: Mater. Sci. Eng. 2017, 169, 012014.

[17] C\&A catalogue, http://www.c-and-a.jp, 24 Feb 2019.

[18] R. Chen, V. Pagonis, Thermally and optically stimulated luminescence: a simulation approach, John Wiley \& Sons, 2011.

[19] A. Nakatsuka, A. Yoshiasa, T. Yamanaka, Acta Crystallogr., Sect. B 1999, 55, 266.

[20] T. Kanai, M. Satoh, I. Miura, J. Am. Ceram. Soc. 2008, 91, 456. 
[21] R. D. Shannon, Acta Crystallogr., Sect. A 1976, 32, 751.

22] S. K. Yadav, B. P. Uberuaga, M. Nikl, C. Jiang,, C. R. Stanek, Phys. Rev. Appl. 2015, 4, 054012.

[23] R.H. Lamoreaux, D. L. Hildenbrand, L. Brewer,J. Phys. Chem. Ref. Data 1987, 16, 419.

[24] K. Kamada, M. Nikl, S. Kurosawa, A. Beitlerova, A. Nagura, Y. Shoji, J. Pejchal, Y. Ohashi, Y. Yokota, A. Yoshikawa, Opt. Mater. 2015, 41, 63.

[25] G. Tamulaitis, A. Vaitkevǐcius, S. Nargelas, R. Augulis, V. Gulbinas, P. Bohacek, M. Nikl, A. Borisevich, A. Fedorov, M. Kor- jik, E. Auffray, Nucl. Instrum. Methods Phys. Res., Sect. A 2017, $870,25$.

[26] M. Korjik, V. Alenkov, A. Borisevich, O. Buzanov, V. Dormenev, G. Dosovitskiy, A. Dosovitskiy, A. Fedorov, D. Kozlov, V. Mechinsky, R. W. Novotny, G. Tamulaitis, V. Vasiliev, H-G. Zaunick, A. A Vaitkevi cius, Nucl. Instrum. Methods Phys. Res., Sect. A 2017, 871, 42.

[27] E. Auffray, R. Augulis, A. Borisevich, V. Gulbinas, A. Fedorov, M. Korjik, M. T. Lucchini, V. Mechinsky, S. Nargelas, E. Songaila, G. Tamulaitis, A. Vaitkevǐcius, S. Zazubovich, J. Lumin. 2016, 178, 54.

Institute of Solid State Physics, University of Latvia as the Center of Excellence has received funding from the European Union's Horizon 2020 Framework Programme H2020-WIDESPREAD-01-2016-2017-TeamingPhase2 under grant agreement No. 739508 , project CAM ART2 